\title{
Intra-Articular Hyaluronan Treatment of Patients with Knee Osteoarthri- tis Waiting for Replacement Surgery
}

\author{
F.J. Blanco*, J.L. Fernández-Sueiro, J.A. Pinto-Tasende, J.C. Fernández-López, M. Ramallal, A. \\ Freire and F. Galdo
}

Osteoarticular and Aging Research Unit, Rheumatology Division, INIBIC-Hospital Universitario A Coruña, Xubias 84, 15006-A Coruña, Spain

\begin{abstract}
Aim: To determine whether hyaluronan (HA) delays and/or reduces the knee replacement surgery (KRS) in patients with osteoarthritis (OA).

Material and Methods: A prospective, single-center, double-blind, randomized, placebo-controlled, pilot clinical trial with two treatment groups [HA (Adant ${ }^{\circledR}$ ) treatment group and placebo treatment group] was conducted. The intra-articular treatments (HA or placebo) consisted of two cycles of five weekly injections with a 24-week interval between each cycle. The efficacy variable was determined by change in pain, articular mobility and functional capacity as measured by WOMAC. All efficacy variables have been analyzed for the intention-to-treat population.

Results: 52 patients (10M/42F) were enrolled in the study (HA group: 26; placebo group: 26). Time until KRS in the HA group subjects (368.8 days) was longer than that in the placebo group (253.9 days). The change in the WOMAC stiffness subscale at 24 weeks, compared to the baseline score, was -22.4 in the HA group and -2.2 in the placebo group $(\mathrm{p}=0.081)$. The change in the physical function WOMAC subscale, compared to the baseline score, was statistically significant at 24 weeks (HA group $=-24.7$ placebo group $=-4.4 \mathrm{p}=0.019$ ). Similar results were found in the change in the total WOMAC index score (HA group $=-23.9$ vs placebo group $=-5.6 \mathrm{p}=0.044$ ).
\end{abstract}

Conclusion: The use of intra-articular HA to treat OA patients on the waiting list for KRS does not delay surgery. However, it could improve the physical condition of patients while they are waiting by surgery.

Keywords: Osteoarthritis, Hyaluronan, Treatment, Knee surgery.

\section{INTRODUCTION}

Osteoarthritis (OA) of the knee is an increasingly common problem due to a more active society, which often leads to predisposing knee injuries, an increasingly elderly population, and a growing percentage of the population that is overweight [1].

The initial management of most patients with OA should be non-operative and may include nonsteroidal antiinflammatory medications (NSAIDs), analgesics, bracing, orthoses, shoe modifications, weight loss, and ambulatory aids (e.g., walking stick held in the opposite hand) [2-5]. Activity modification may also be necessary. Home health care-assistive devices for daily living (e.g., toilet extenders, safety rails, and bath seats) may help the patient cope with disability and should be prescribed after consultation with the physical therapist. Knee rehabilitation under the supervision of a physiotherapist may include strengthening and range-of-motion exercises, gait training, and patient education. The patient may undergo intra-articular injection in the knee with steroids or viscosupplementation with hyaluronan (HA) [6-14].

\footnotetext{
*Address correspondence to this author at the Laboratorio de Investigación Osteoarticular, Unidad de Investigación, Hospital Universitario A Coruña., C/ Xubias, 84, 15006-A Coruña, Spain; Tel: 34-981-178272; Fax: 34-981178273; E-mail: fblagar@canalejo.org
}

Responses to non-operative treatment, however, are varied and unpredictable. The progressive nature of the disease means that many patients with OA of the knee eventually require operative treatment [15]. Most patients seem satisfied with their knee replacements, and if pain relief is the main indication for surgery, this indeed should be the case. Satisfactory knee function is usually restored following knee replacements surgery and the majority of patients are able to return to low-impact sporting activity.

The Spanish Health System is public, and all patients can therefore undergo a free knee replacement surgery (without any economic cost for patient) after a correct medical indication. For this reason, many OA patients are included on a waiting list. The time that a patient stays on the waiting list varies and is dependent on the specific hospital. Most patients who undergo knee replacement surgery are elderly with co-morbid diseases that put them at high risk to undergo surgery. Outcomes of knee replacement surgery are dependent on several variables, such as the timing of the surgery and the number of times the surgeon and the hospital have performed the procedure. Furthermore, the patient's preoperative medical status, peri- and postoperative management, and rehabilitation play important roles in recuperation [16].

The original rationale for the use of intra-articular HA to treat $\mathrm{OA}$ was to increase the viscosity of the synovial fluid. Hyaluronan is a heteropolysaccharide comprised of a variable number of repeating units of D-glucuronic acid and $\mathrm{N}$ - 
acetylglucosamine [17]. Synoviocytes, fibroblasts and chondrocytes all synthesize HA, which is present in synovial fluid and the extracellular matrix of cartilage [18]. Because HA is viscoelastic, it behaves as a viscous liquid at low shear rates and as an elastic solid at high shear rates. Intra-articular injections of HA are used to treat OA to reduce joint pain. For this reason, HA is indicated to treat knee OA grades II-III [25]. However, no studies have been performed in knee OA grade IV or in OA patients who have been given an indication for surgery. Taking into account this lack of data, the main objective of this study was to assess if treatment with HA, compared to treatment with a placebo, may delay and/or reduce the knee replacement surgery. The secondary objective of the study was to study whether the intra-articular administration of HA in knee OA patients reduces pain and improves functional capacity, and to assess the safety of the treatments.

\section{MATERIALS AND METHODS}

\section{Study Design and Population}

A prospective, double-blind, randomized, placebo-controlled, single-center, outpatient pilot clinical trial was conducted with subjects who were included on the waiting list of Hospital Universitario A Coruña for knee replacement surgery. These subjects were experiencing severe pain, quantified by the WOMAC (Western Ontario Mc Master University) OA Index, index pain subscale $(\geq 150 \mathrm{~mm})$, despite long-term treatment with NSAIDs and analgesics. Subjects were included in two treatment groups: $900 \mathrm{kDa} H A$ (Adant ${ }^{\circledR}$, Tedec-Meiji Farma, S.A.) treatment group and placebo treatment group. Treatment with rescue analgesics (paracetamol $4000 \mathrm{mg} / \mathrm{day}$ ) and/or NSAIDs (Diclofenac 150 $\mathrm{mg} /$ day) was permitted in all subjects. The consumption of paracetamol and diclofenac was quantified and recorded in each visit. The intra-articular treatments (HA or placebo) consisted of two cycles of five weekly injections with a 24week interval between each cycle. The patients were assigned to one of the two groups using a randomization list. Subjects were evaluated by telephone follow-up one week after each cycle and by visits 12 weeks (V6 and V13) and 24 weeks (V7 and V14) after each cycle (Fig. 1).

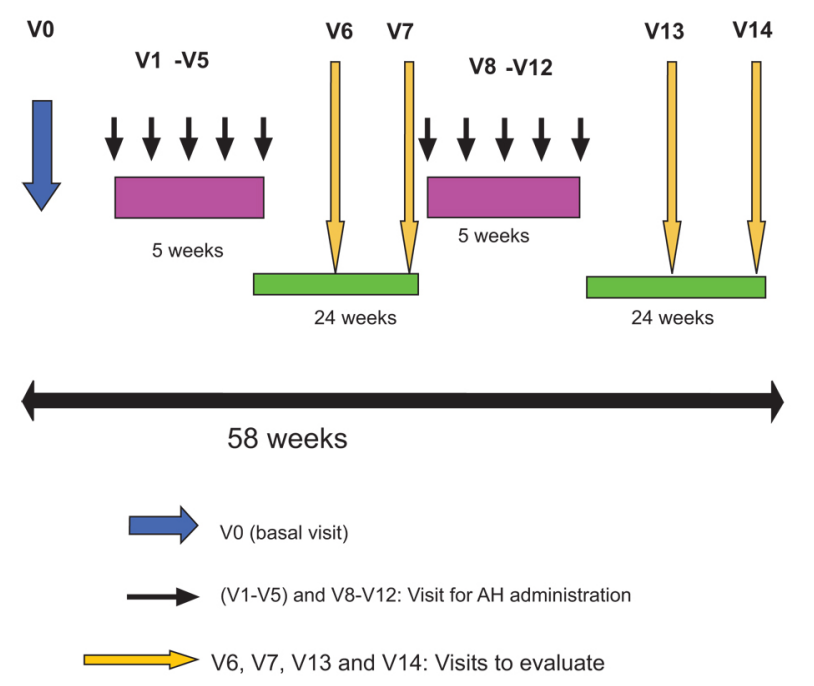

Fig. (1). Scheme of visits and treatment.
Prior to each injection, synovial fluid, if present, was first aspirated and then $2.5 \mathrm{ml}$ of $\mathrm{HA}(25 \mathrm{mg})$ in saline in the HA group or $2.5 \mathrm{ml}$ of saline vehicle (without $\mathrm{HA}$ ) in the placebo group was injected into the study knee at weekly intervals for five weeks (Cycle 1: V1-V5; Cycle 2: V8-V12) (Fig. 1). Subjects over 40 years of age without joint inflammation were selected for this study if symptomatic OA was evidenced by pain according to the American College of Rheumatology (ACR) criteria [19] and if they were grade IV using the Kellgren-Lawrence (K-L) scoring [20]. Patients were excluded from this study if they had received intra-articular injections of corticosteroids in the target joints within three months of study entry or HA injections within one year of study entry. Patients who had received glucosamine sulphate during the three months prior to beginning the study or had used an investigational drug within 30 days of study entry or during the study schedule were excluded. Individuals with previous knee surgery that would interfere with the evaluation of the results of this study or who had a history of rheumatoid arthritis, ankylosing spondylitis, microcrystalline arthropathies, chondrocalcinosis, fibromyalgia or any other pathology of the knee that could interfere with the study and assessments were also excluded. Other exclusion criteria were patients with severely impaired central nervous systems, impaired coagulation, known sensitivity to HA, paracetamol or diclofenac, or were immuno-compromised, receiving systemic inmuno-suppressive therapy, or considered by the investigator to be unable to complete the treatment or follow-up.

Subjects were recruited from within the investigators' medical practices. Subjects provided written consent to participate. The study was designed in compliance with the Declaration of Helsinki and successive reviews. Regional Committee of Clinical Investigation (CEIC of Galicia-Spain) approved the study protocol. The study required both a blinded evaluator and an unblinded administrator. The blinded evaluator performed and recorded efficacy assessments and was not present during the injection or given access to other post-baseline study data (e.g., adverse event and laboratory data). The unblinded administrator recorded adverse events and was responsible for administering the injections (HA and placebo injections had the same external appearance). Neither the patient nor the blinded evaluator could determine to which therapeutic group the patient was assigned.

Prospective follow-up of the patients of study was performed after 1 year.

\section{Outcome Measures}

The primary objective was to analyze the efficacy of the treatment based on whether intra-articular HA treatment delayed the time of knee replacement surgery or reduced the number of knee replacement surgeries in OA patients on the waiting list for knee surgery at Hospital Universitario A Coruña. This delay would be the result of an improvement in the signs and symptoms of OA. This improvement was assessed by the WOMAC OA Index questionnaire scores [21], a multidimensional measure of pain, stiffness, and physical functional disability comprised of 24 questions and an overall score. Items are scored by a visual analogue scale (VAS) from 0 (no pain, symptoms, or physical disability) to 100 
$\mathrm{mm}$ (extreme levels). Each subscale was transformed to a range from zero to 100 points, a score of 100 indicating best condition and 100 the worst condition.

Efficacy analyses were performed on the intention-totreat (ITT) population, defined as all randomized subjects who took at least one dose of study medication and for whom a post-randomization efficacy measurement was available. Lack of efficacy was indicated by discontinuation due to insufficient pain relief.

Safety was also monitored throughout the study. Assessments were performed on randomized subjects who were administered at least one dose of study medication and had at least one available post-baseline safety measurement. The number and percentage of subjects reporting adverse events and their severity were tabulated for both treatment groups, and subjects reporting serious adverse events or withdrawing due to an adverse event were recorded. An assessment of the relationship of adverse events to study medication was also conducted. Naranjo's algorithm was used to determine the degree of causality [22].

\section{Statistical Analyses}

Three different subject populations were defined. The intention-to-treat population (ITT) includes all randomized subjects that received at least one study treatment or placebo injection and reached at least one efficacy assessment visit, regardless of whether they complied with the treatment they were given. The per protocol population (PP) includes all randomized subjects complying adequately with the protocol criteria. The safety population (SAF) includes all randomized subjects that received at least one study treatment injection and from whom any safety data was obtained during the follow-up.

To analyze the effect of the treatment on time to knee replacement surgery, the method of Kaplan-Maier curves was employed. If appropriate, the $95 \%$ confidence interval of median time to knee surgery was calculated. This analysis was performed in all patients of both intra-articular groups (HA and placebo). A Log-Rank test was used in order to compare both survival (until knee replacement surgery) functions. The time to knee replacement surgery was defined as

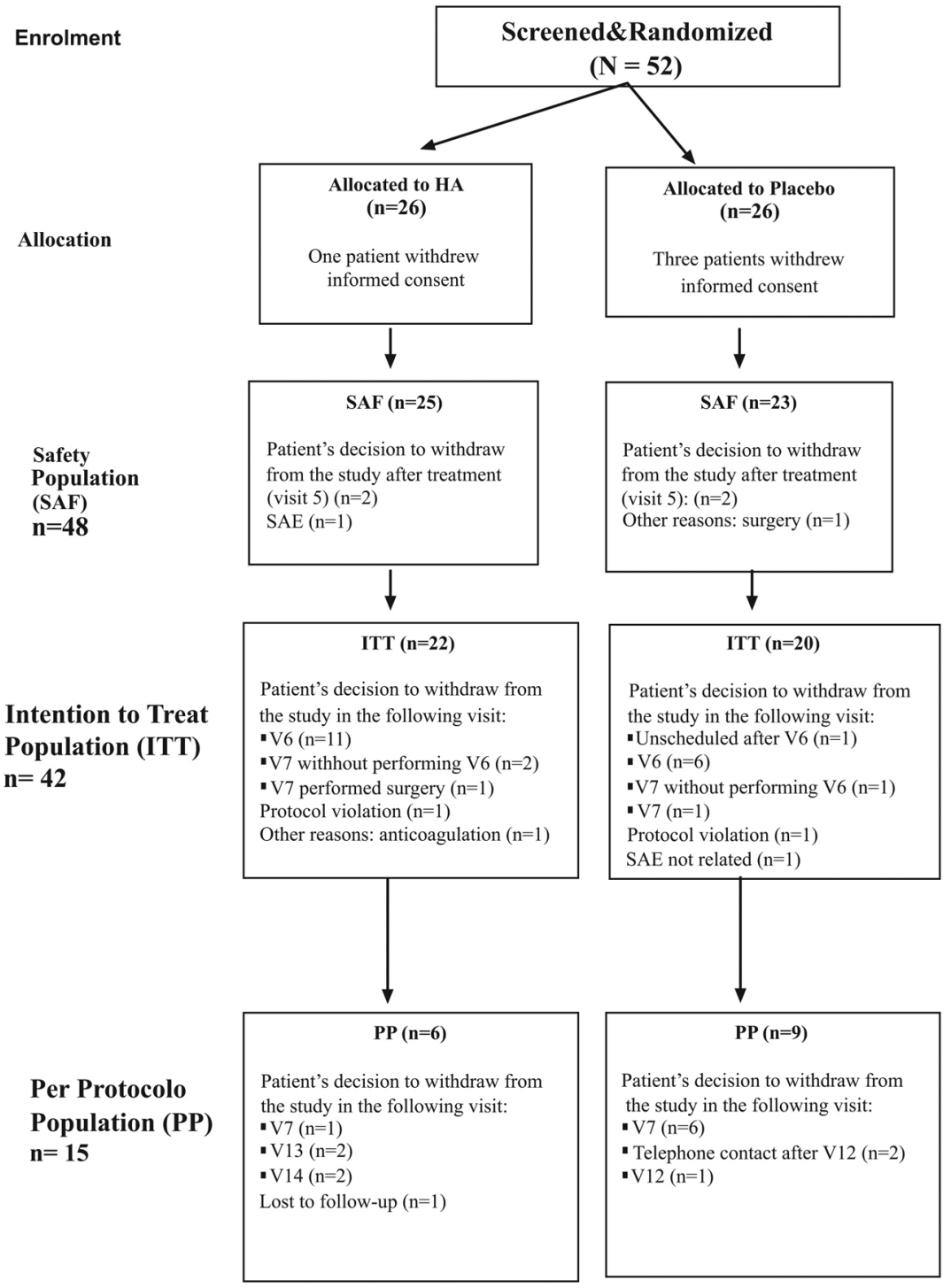

Fig. (2). Subject disposition. 
the number of days between the randomization date and the date of knee surgery. Patients without knee surgery replacement were assigned the time of the last assessment for knee surgery. Patients who discontinued the study for any reason without knee surgery or for whom no follow-up information on knee replacement surgery was available, were assigned the time when the subject was last known not to have received knee replacement surgery. Additionally, The ManWhitney Wilcoxon U test was used to detect any statistically significant differences in time to knee replacement surgery. This test was performed in patients within the intra-articular groups who had received surgery.

For efficacy measures the statistical significance of any difference in the mean scores between the HA and placebo groups was assessed by the Student-t test or the MannWhitney Wilcoxon U-test.

\section{RESULTS}

Fifty-two subjects were included and randomized (Placebo: $n=26$; HA: $n=26$ ) into this study. Four patients withdrawn their consent and were not administered treatment therefore, the safety population (SAF) consisted of $88.5 \%$ $(n=23)$ of the placebo subjects and $96.2 \%(n=25)$ of the HA subjects. Furthermore, $76.9 \%$ from the placebo group $(\mathrm{n}=$ $20)$ and $84.6 \%(n=22)$ from the HA group were included in the intention-to-treat population (ITT). The per-protocol population (patients whose completed 58 weeks of study) included $34.6 \%(\mathrm{n}=9)$ from the placebo subjects and $23.1 \%$ $(n=6)$ from the HA subjects. The disposition of subjects as well as the causes to withdraw from the clinical trial is shown in Fig. (2).

A summary of demographic and baseline characteristics of the ITT population is presented in Table $\mathbf{1}$. There were no statistically significant differences $(p>0.05)$ between the treatment groups with respect to baseline demographics and other variables studied. The mean baseline WOMAC scores for each category (pain, stiffness, physical function and overall) were also similar for both treatment groups and without statistical differences (Table 1).

For the ITT population, subjects in the HA group had a significantly improved, (lower) mean total WOMAC index score at 24 weeks compared to the placebo, with statistical significance $(\mathrm{HA}$ group $=-23.9$ vs placebo group $=-5.6 \mathrm{p}=$ 0.044) (Table 2). In addition, subjects treated with HA also had an improved mean final WOMAC scale for physical function compared with the placebo group subjects (HA group $=-24.7$ vs placebo group $=-4.4 \mathrm{p}=0.019$ (Table 2) Furthermore, although no statistical differences were found between the two treatment groups, it can be observed that the HA group subjects improved in the pain and stiffness subscale analyzed at 24 weeks post-first cycle of treatment by WOMAC (Table 2).

In the subjects treated with HA, the mean percentage of variation in WOMAC subscale of pain was $-17.3 \%$, with a $57.1 \%$ of patients experiencing reduction. In addition, in those patients treated with HA who rejected surgery, $75 \%$ showed decreases in the WOMAC pain subscale at 24 weeks. In these patients the mean percentage of decrease for the WOMAC pain subscale was $-26.3 \%$. Interestingly, the percentages of patients per group that use permitted rescue medication were only $47.8 \%$ and $56.0 \%$ for placebo and HA groups respectively $(\mathrm{p}=0.571)$.

Prospective follow-up after 1 year was performed in all patients. Survival analysis showed that, although there was not statistical significance, survival functions differed. Survival time until knee replacement surgery in the HA group subjects (368.8 days) was higher than that in the placebo group subjects (253.9 days) (Table 3). However, the LogRank test did not show statistical differences between the survival functions $(\mathrm{p}=0.249$ ) (Fig. 2). Furthermore, the pro-

Table 1. Demographic and Baseline Data for Intention-to-Treat Population

\begin{tabular}{|c|c|c|}
\hline Variable & $\begin{array}{c}\text { Placebo } \\
\mathbf{N}=\mathbf{2 0}\end{array}$ & $\begin{array}{c}\text { HA } \\
\mathbf{N}=\mathbf{2 2}\end{array}$ \\
\hline $\begin{array}{c}\text { Gender } \\
(\text { males })^{\mathrm{a}} \\
\text { (females) }^{\mathrm{a}}\end{array}$ & $\begin{array}{c}5(25.0 \%) \\
15(75.0 \%)\end{array}$ & $\begin{array}{c}5(22.7 \%) \\
17(77.3 \%)\end{array}$ \\
\hline $\begin{array}{c}\text { Ethnic } \\
\text { (Caucasian) }^{\text {a }}\end{array}$ & $20(100.0 \%)$ & $22(100.0 \%)$ \\
\hline Age (years) ${ }^{b}$ & $68.3(9.1)$ & $67.5(8.1)$ \\
\hline Body Mass Index (BMI) $(\mathrm{Kg} / \mathrm{m} 2)^{\mathrm{b}}$ & $34.2(6.7)$ & $32.7(4.1)$ \\
\hline Years since diagnosis $^{c}$ & $11(11.0)$ & $10.0(7.0)$ \\
\hline Pain WOMAC subscale ${ }^{\mathrm{d}}$ & $67.6(60.4-74.8)$ & $62.6(58.9-66.4)$ \\
\hline Total WOMAC index score ${ }^{\mathrm{d}}$ & $70.1(63.2-77.1)$ & $63.1(56.4-69.9)$ \\
\hline
\end{tabular}

${ }^{\mathrm{a}} \mathrm{n}(\%)$.

${ }^{\mathrm{b}}$ Mean (S.D.).

${ }^{\mathrm{c}}$ Median (interquantilic range).

${ }^{\mathrm{d}}$ Mean $(95 \% \mathrm{CI})$. 
Table 2. Change at 6 Months in Total WOMAC Index and in WOMAC Subscale

\begin{tabular}{|c|c|c|c|}
\hline & $\begin{array}{c}\text { Placebo n= 20 } \\
\text { Mean (SD) }\end{array}$ & $\begin{array}{c}\text { Hyaluronan n=22 } \\
\text { Mean (SD) }\end{array}$ & p value \\
\hline \hline Total WOMAC index & $-5.6(21.2)$ & $-23.9(17.9)$ & 0.044 \\
\hline Pain WOMAC subscale & $-11.2(21.0)$ & $-21.7(25.9)$ & 0.307 \\
\hline Stiffness WOMAC subscale & $-2.2(40.3)$ & $-22.4(22.5)$ & 0.081 \\
\hline Functional capacity WOMAC subscale & $-4.4(18.8)$ & $-24.7(18)$ & 0.019 \\
\hline
\end{tabular}

$\mathrm{p}$ value was calculated using Mann-Whitney Wilcoxon test.

Table 3. Survival Time to Surgery

\begin{tabular}{|c|c|c|}
\hline & $\begin{array}{c}\text { Placebo } \\
\mathbf{n = 2 3}\end{array}$ & $\begin{array}{c}\text { Hyaluronan } \\
\mathbf{n = 2 5}\end{array}$ \\
\hline \hline Number of patients with surgery & 20 & 16 \\
\hline Number of patients without surgery & 3 & 9 \\
\hline Days to surgery & 253.8 & 368.7 \\
(Mean-IC 95\%) & $(165-314)$ & $(185-)$ \\
\hline
\end{tabular}

MannWithney Wilconxon test: $\mathrm{p}=0.186$.

portion of subjects discontinuing treatment at 24 weeks due to lack of efficacy was higher in the placebo group (20/23, $87 \%)$ compared with the HA group $(16 / 25,64 \%)(p=0.06)$ (Fig. 3). Knee surgery was avoided in 9 and 3 patients from $\mathrm{HA}$ and placebo groups respectively (Table $\mathbf{3}$ ).

A total of 48 patients (placebo group $=23$ and HA group $=25$ ) were evaluated for safety (SAF population). Only AE that occurred after the first injection of HA or placebo have been taken into account. Thus, $34.8 \%$ and $16.0 \%$ of patients in the placebo and HA groups respectively reported at least one AE (abdominal pain $8.7 \%$, insomnia 8.7\%). There were no significant differences between groups in the number of AEs. Related adverse events (AE) did not occur in either the placebo group or the HA group.

\section{DISCUSSION}

Available therapies for managing OA provide only symptomatic relief. Currently, exogenous HA administered as an intra-articular injection is an option to treat $\mathrm{K}-\mathrm{L}$ grade II/III knee OA [6-14]. However the efficacy of HA treatment in OA patients with a K-L grade IV is unknown. Given the absence of information in this OA population, we designed this pilot clinical trial. The main objective was to assess if treatment with HA, compared to treatment with a placebo, delays and/or reduces the knee replacement surgery.

To implement this clinical trial we selected knee OA patients on the waiting list for knee replacement surgery. Because variations in waiting times are associated with the sys-

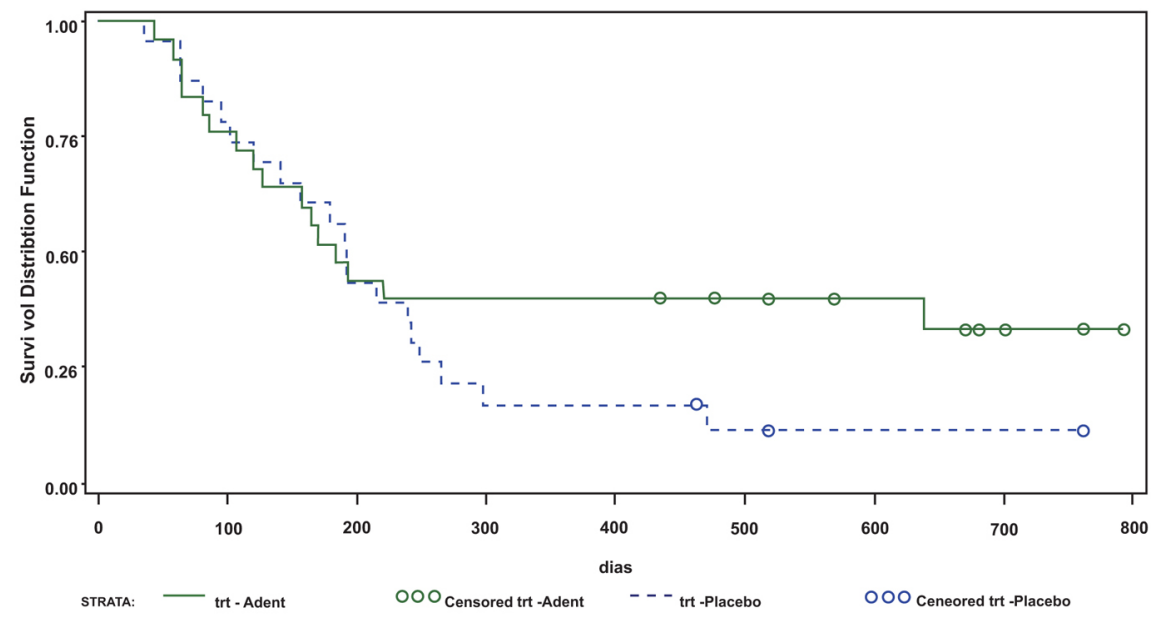

Log-Rank test $\mathrm{p}=0.249$

Fig. (3). Kaplan Maier curve showing evolution of patients treated with Hyaluronan and with placebo. 
tem of reimbursement for surgeons and whether the hospital is publicly funded or private [23], we decided (i) to perform the clinical trial in a public hospital (Hospital Universitario A Coruña) and, (ii) that the indication for surgery would always be done by the same surgeon (MR). Indication to knee surgery was severe pain at rest or nocturnal pain, as well as important limitation of patient to perform daily activity.

All subjects included in the study were experiencing severe pain, as shown by the baseline values on the WOMAC pain subscale for both treatment groups. Interestingly, the percentage of subjects in each treatment group that used permitted rescue medications during the study was very low (around 50\% in both groups). Explanations given for the low amounts of analgesics used by the subjects were that the medications were not efficacious and that the subjects were afraid of the side effects of long-term medications. Generally the subjects took rescue medications only when they had planned extra activities, such as visits to the home of friends or family and "going out to dinner". These subjects were able to perform the normal activities of daily living without taking medication.

The most common reason for discontinuation in the study was subject decision, $82.6 \%$ and $84 \%$ for placebo and $\mathrm{HA}$, respectively. However, the total percentage of subject compliance for cycle 1 (those subjects who had received all the HA and placebo injections according to the protocol for cycle 1) was $95.7 \%$ and $100 \%$ for the placebo and HA groups, respectively. The total percentage of subject compliance for cycle 2 (those subjects who had received all the HA or placebo injections according to the protocol) was $13 \%$ and $20 \%$ for the placebo and HA groups, respectively.

Taking into account the limited number of patients included, this pilot study cannot provide robust evidence that HA injections are better than placebo injections for treating patients with K-G grade IV knee OA. However, this pilot study does have important data for the future treatment of patients prior to knee replacement surgery. The HA group subjects appear to have a good efficacy profile at six months. In some subjects receiving intra-articular injections surgery was avoided ( 3 patients in placebo group and 9 patients in HA group). Prospective follow-up of these patients showed that after 1 year, 2 patients from placebo group and none patients from HA group were submitted to surgery.

In addition, the subjects receiving HA treatment improved their general condition while they were on the waiting list. These patients showed a numerical, although not statistically significant, improvement in knee pain on the scale used. Although the differences were not significant, about $57 \%$ of subjects from the HA group showed a reduction in knee pain. We must keep in mind that analgesics and NSAIDs were permitted throughout the clinical trial and patients on a waiting list for knee surgery can overestimate the pain [24]. The clinical significance of these findings is supported by secondary outcomes that measured physical function, total WOMAC index score, WOMAC physical functional and WOMAC subscale for stiffness. Because patients on a waiting list for knee surgery have an important disability, minimal changes improving physical function may have a large effect on their activities of daily living [25].
Because articular function prior to knee replacement surgery is important for improving the post-surgical outcome [26], the administration of intra-articular HA to patients on a waiting list may be considered as an alternative treatment. Therefore, the results of our study can be considered clinically relevant.

In addition to efficacy this clinical trial confirms that the intra-articular injection of HA is safe. Throughout the course of this study, 150 injections of HA were given and the safety evaluations of the subjects were excellent.

A possible explanation for these results lies in the mechanism of action of HA. In OA, the concentration of synovial fluid HA is reduced, the length of the HA chains is decreased, and the viscoelastic properties of the synovial fluid are compromised $[27,28]$. HA is viscoelastic and behaves as a viscous liquid at low shear rates and as an elastic solid at high shear rates. Although the original rationale for the use of intra-articular injections of HA in OA was to increase the viscosity of the synovial fluid, HA also has a variety of cellular effects including: 1) inhibition of prostaglandin E2 (PGE2) and nitric oxide (NO) synthesis induced by IL-1 (interleukin-1); 2) protection against proteoglycan depletion; 3) protection against cytotoxicity induced by oxygen-derived free radicals and against apoptosis induced by NO and Fas stimulation; 4) modulation of leukocyte adherence, proliferation, migration and phagocytosis; and, 5) suppression of cartilage matrix degradation by fibronectin fragments [29-34]. HA is able to induce some of these biological effects on synoviocytes and fibroblasts, causing a reduction of pro-inflammatory mediators and increased HA concentration in the joint. Taken together, these could cause a reduction in joint stiffness with improvement in the physical function of the joint.

In summary, this pilot clinical trial suggests that the use of intra-articular HA to treat OA patients on the waiting list for KRS does not delay significantly the surgery. However, treatment with HA could significantly improve the physical condition of the patients because it reduces significantly the WOMAC total score and the physical function WOMAC subscale. These results would need to be confirmed with a larger study.

\section{ACKNOWLEDGEMENTS}

We are grateful to Ms. Pilar Cal Purriños for her expert secretarial assistance.

\section{FINANCIAL SUPPORT}

This study was supported by a grant from Tedec-Meiji Farma, S.A. Carlos Fernández-Lopez was supported by Fondo de Investigación Sanitaria, Programa Post-MIR.

\section{REFERENCES}

[1] Blanco FJ, Fernández-López JC, Galdo-Fernández F. Artrosis. Medicine. Barcelona, 2004; 1: 1-12.

[2] Altman RD, Hochberg MC, Moskowitz RW. Recommendations for the medical management of osteoarthritis of the hip and knee. 2000 update. American College of Rheumatology Subcommittee on Osteoarthritis guidelines. Arthritis Rheum 2000; 43(9): 1905-15.

[3] Hochberg MC, Altman RD, Brandt KD, et al. Guidelines for the medical management of osteoarthritis. Arthritis Rheum 1995; 38 (11): 1535-46.

[4] Panel de Expertos de la Sociedad Española de Reumatología. Primer documento consenso de la Sociedad Española de Reumatolo- 
gía para el tratamiento farmacológico de la artrosis de rodilla. Reumatol Clin 2005; 1: 38-48.

[5] Zhang W, Doherty M, Arden N, et al. EULAR Standing Committee for International Clinical Studies Including Therapeutics (ESCISIT). EULAR evidence based recommendations for the management of hip osteoarthritis: report of a task force of the EULAR Standing Committee for International Clinical Studies Including Therapeutics (ESCISIT). Ann Rheum Dis 2005; 64: 669-81.

[6] Altman RD, Moskowitz RW. Hyalgan ${ }^{\circledR}$ Study Group. Intraarticular sodium hyaluronate (Hyalgan $\left.{ }^{\circledR}\right)$ in the treatment of patients with osteoarthritis of the knee: A randomized clinical trial. J Rheumatol 1998; 25: 2203-12.

[7] Creamer P. Intraarticular corticosteroid injections in osteoarthritis: Do they work and if so, how?. Ann Rheum Dis 1997; 56: 634-6.

[8] Dieppe PA. Are intraarticular steroid injections useful for the treatment of the osteoarthritis joint? Br J Rheumatol 1991; 30: 199.

[9] Namiki O, Toyoshima H, Morisaki N. Therapeutic effect of intraarticular injection of high molecular weight hyaluronic acid on osteoarthritis of the knee. Int J Clin Pharmacol Ther Toxicol 1982; 20: 501-7.

[10] Peyron JG, Balazs EA. Preliminary clinical assessment of Nahyaluronate injection into human arthritic joints. Pathol Biol 1974; 22: 731-6.

[11] Bellamy N, Campbell J, Robinson V, Gee T, Bourne R, Wells G. Viscosupplementation for the treatment of osteoarthritis of the knee. Cochrane Database Syst Rev 2005; (2): CD005321.

[12] Bellamy N, Campbell J, Robinson V, Gee T, Bourne R, Wells G. Intraarticular corticosteroid for treatment of osteoarthritis of the knee. Cochrane Database Syst Rev 2005; (2): CD005328.

[13] Ghosh P, Guidolin D. Potential mechanism of action of intraarticular hyaluronan therapy in osteoarthritis: Are the effects molecular weight dependent? Semin Arthritis Rheum 2002; 32: 10-37.

[14] Day R, Brooks P, Conaghan PG, Petersen M. Multicenter Trial Group. A double blind, randomized, multicenter, parallel group study of the effectiveness and tolerance of intraarticular hyaluronan in osteoarthritis of the knee. J Rheumatol 2004; 31: 775-82.

[15] Pagnano MW, Clarke HD, Jacofsky DJ, Amendola A, Repicci JA. Surgical treatment of the middle-aged patient with arthritic knees. Instr Course Lect 2005; 54: 251-9.

[16] Jones CA, Beaupre LA, Johnston DW, Suarez-Almazor ME. Total joint arthroplasties: Current concepts of patient outcomes after surgery. Clin Geriatr Med 2005; 21(3): 527-41.

[17] Jackson RL, Busch SJ, Cardin AD. Glycosaminoglycans: molecular properties, protein interactions and role in physiological processes. Physiol Rev 1991; 71: 481-538.

[18] Smith MM, Gosh P. The synthesis of hyaluronic acid by human synovial fibroblasts is influenced by the nature of the hyaluronate in the extracellular environment. Rheumatol Int 1987; 7: 113-22.

[19] Altman RD, Asch E, Bloch D, et al. Development of criteria for the classification and reporting of osteoarthritis. Classification of Osteoarthritis of the knee. Arthritis Rheum 1986; 29 (8): 1039-49.

[20] Kellgren JH, Lawrence JS. Radiological assessment of osteoarthritis. Ann Rheum Dis 1957; 16: 494-501.
[21] Bellamy N, Buchanan WW, Goldsmith $\mathrm{CH}$, et al. Validation study of WOMAC: A health status instrument for measuring clinically important patient relevant outcomes to antirheumatic drug therapy in patients with osteoathritis of the hip or knee. J Rheumatol 1988 15: $1833-40$

[22] Naranjo CA, Bustos V, Sellers EM, et al. A method for estimating the probability of adverse drug reactions. Clin Pharmacol Ther 1981; 30: 239-45.

[23] Lingard EA, Berven S, Katz JN. Kinemax Outcomes Group. Management and care of patients undergoing total knee arthroplasty: Variations across different health care settings. Arthritis Care Res 2000; 13: 129-36.

[24] Hawker GA, Wright JG, Badley EM, Coyte PC. Perceptions of, and willingness to consider, total joint arthroplasty in a populationbased cohort of individuals with disabling hip and knee arthritis. Arthritis Rheum 2004; 51: 635-41.

[25] Kelly KD, Voaklander DC, Johnston DW, Newman SC, SuarezAlmazor ME. Change in pain and function while waiting for major joint arthroplasty. J Arthroplasty 2001; 16: 351-9.

[26] Berge DJ, Dolin SJ, Williams AC, Harman R. Pre-operative and post-operative effect of a pain management programme prior to total hip replacement: A randomized controlled trial. Pain 2004; 110: 33-9.

[27] Balazs EA, Watson D, Duff IF, Roseman S. Hyaluronic acid in synovial fluid. Molecular parameters of hyaluronic acid in normal and arthritis human fluids. Arthritis Rheum 1967; 10: 357-76.

[28] Dahl LB, Dahl IMS, Engstrom-Laurent A, Granath K. Concentration and molecular weight of sodium hyaluronate in synovial fluid from patients with rheumatoid arthritis and other arthropathies. Ann Rheum Dis 1985; 44: 817-22.

[29] Ghosh P. The role of hyaluronic acid in health and disease: interactions with cells, cartilage and components of the synovial fluid. Clin Exp Rheumatol 1994; 12: 75-82.

[30] Yasui T, Adatsuka M, Tobetto K, Hayaishi M, Anto T. The effect of hyaluronan on IL-1 induce PGE2 production in human OA synovial cells. Agents Actions 1992; 37: 155-6.

[31] Lisignoli G, Grassi F, Zini N, et al. Anti-Fas-induced apoptosis in chondrocytes reduced by hyaluronan. Arthritis Rheum 2001; 44 1800-7.

[32] Takahashi K, Hashimoto S, Kubo T, Hirasawa Y, Lotz M, Amiel D. Effect of hyaluronan on chondrocyte apoptosis and nitric oxide production in experimentally induced osteoarthritis. J Rheumatol 2000; 27: 1713-20.

[33] Presti D, Scott JE. Hyaluronan mediated protective effect against cell damage caused by enzymatically generated hydroxyl radicals is dependent on hyaluronan molecular mass. Cell Biochem Funct 1994; $12: 281-8$

[34] Maneiro E, de Andres MC, Fernandez-Sueiro JL, Galdo F, Blanco FJ. The biological action of hyaluronan on human osteoartritic articular chondrocytes: The importance of molecular weight. Clin Exp Rheumatol 2004; 22: 307-12. 